\title{
Antimicrobial Sensitivity Pattern of Pseudomonas Aeruginosa Isolated from a Tertiary Care Hospital
}

\author{
Sabita Bhatta, Manoj Pradhan, Alina Singh, Raina Chaudhary and Yankpam Ibotomba Singh
}

Department of Clinical Microbiology, Nepalese Army Institute of Health Sciences, Shree Birendra Hospital, Chhauni, Kathmandu, Nepal

\begin{abstract}
Introduction: Pseudomonas aeruginosa ( $P$. aeruginosa) is one of the major cause of nosocomial infection. Multi-drug-resistant $P$. aeruginosa (MDR PA) are increasingly encountered in clinical samples .Therefore, this study was undertaken to know the sensitivity pattern of $P$. aeruginosa and to detect MDR PA from clinical samples.

Methods: This was a laboratory based retrospective-cross sectional study conducted at Department of Microbiology at a tertiary care referral centre in Kathmandu, Nepal from December 2018 to December 2019. Total of 200 isolates of $P$. aeruginosa were isolated from clinical samples. Non repetitive sample per patient was included in the study. Samples were processed according to standard methodology and antimicrobial sensitivity testing (AMST) was carried out by Modified Kirby Bauer disk diffusion test as per Clinical Laboratory Standard Institute guideline. MDR was defined as strains resistant to one or more agent of $\geq 3$ groups of antimicrobial categories.

Results: Highest number of $P$. aeruginosa were isolated from sputum (93), followed by wound swab (35), and pus (29). AMST revealed the most sensitive drug to be Amikacin (91.51\%) followed by Meropenem (78.5\%) and Piperacillin/Tazobactam (77.50\%). Out of 200 isolates, 69 (34.5\%) were MDRPA.

Conclusions: The information regarding sensitivity pattern of $P$. aeruginosa will help clinicians to choose most effective antimicrobials for the treatment of patients thus saving the time duration and total cost of patient management and also it will play a key role in setting antimicrobial stewardship policy.
\end{abstract}

Key words: antimicrobial sensitivity; multidrug resistant; Pseudomonas aeruginosa

Correspondence: Sabita Bhatta, Department of Clinical Microbiology, Nepalese Army Institute of Health Sciences, Shree Birendra Hospital, Chhauni, Kathmandu, Nepal. E-mail: bhattasabita@gmail.com

DOI: http://dx.doi.org/10.3126/mjsbh.v19i2.28380

Submitted on: 2020-04-09

Accepted on: 2020-05-09

This work is licensed under creative common license:

http://creativecommons.org/licenses/by-nc-nd/4.0/ (C) MJSBH 2020 


\section{INTRODUCTION}

Pseudomonas aeruginosa is the major pathogenic species in the family Pseudomonadaceae. The ability of $P$. aeruginosa to grow with minimal nutrient requirement and its tolerance to diverse conditions has allowed this organism to persist in hospital as well as in community. Outside the hospital, $P$. aeruginosa is commonly found in soil, water and plants. Within the hospital, $P$. aeruginosa can colonize moist surfaces of patient, moist inanimate objects in the environment including water in sinks, drains, toilet, showers, mops, respiratory ventilators and cleaning solutions. ${ }^{1,2}$

$P$. aeruginosa emerged as a major human pathogen in the $1960 \mathrm{~s}$, since than it has become one of the most serious cause of nosocomial bacterial infections. ${ }^{3,4}$ Furthermore, the increasing frequency of MDRPA are increasingly encountered in clinical samples, leaving clinicians with very few effective drugs for treatment of patient leading to high morbidity and mortality.

Many studies have shown that treatment of MDR PA infections are really challenging with huge financial burden. Therefore, this study was carried out with the objective to isolate $P$. aeruginosa from various clinical samples, to know their antimicrobial sensitivity pattern and to identify multi drug resistant pseudomonas aeruginosa (MDRPA) among the isolates which will ultimately help clinicians to choose better treatment option.

Table 1. Distribution of $P$. aeruginosa in clinical samples

\begin{tabular}{|l|r|r|}
\hline Sample type & $\begin{array}{l}\text { Number of } \\
\text { isolates }\end{array}$ & \% of isolates \\
\hline Sputum & 93 & 46.5 \\
Wound swab & 35 & 17.5 \\
Pus & 29 & 14.5 \\
Tracheal aspirate & 20 & 10 \\
Urine & 10 & 5 \\
Throat swab & 5 & 2.5 \\
Bronchoalveolar & 5 & 2.5 \\
lavage & & 1 \\
Eye swab & 2 & 0.5 \\
Pleural fluid & 1 & \\
\hline
\end{tabular}

\section{METHODS}

This was a laboratory based retrospective-cross sectional study carried out at department of Microbiology in a teaching institute located in Kathmandu, Nepal from 2018 December to 2019 December. Total of 200 P. aeruginosa were isolated from various clinical samples. Non repetitive sample per patient was included in the study. Antimicrobial sensitivity testing was carried by Modified Kirby Bauer disk diffusion method. Interpretation was done as per Clinical and Laboratory Standards Institute (CLSI) guideline. ${ }^{5}$ The definition of MDR was established as isolates resistant to at least one or more agent of $\geq 3$ groups of antimicrobial categories. ${ }^{6}$ P. aeruginosa ATCC 27853 was used as quality control.

\section{RESULT}

Total of $200 P$. aeruginosa were isolated from various clinical samples. The details of sample wise distribution is shown in Table 1. Antimicrobial sensitivity testing revealed Amikacin $(91.5 \%)$ to be the most sensitive drug against $P$. aeruginosa followed by Meropenem which is depicted in Table 2. Out of 200 isolates, 69 (34.5\%) were MDR PA.

\section{DISCUSSION}

$P$. aeruginosa, particularly MDRPA has become challenge for the treatment leading to high morbidity and mortality. P. aeruginosa was most commonly isolated from upper and lower respiratory tract followed by pus and wound swabs as reported by Agrawal $\mathrm{S}$ et al. and Ansari $\mathrm{S}$ et al. ${ }^{7,8}$ In this study also, highest number of $P$. aeruginosa was isolated from upper and lower respiratory tract

Table 2. Sensitivity Pattern of $P$. aeruginosa $(\mathrm{n}=200)$

\begin{tabular}{|l|r|r|}
\hline Antibiotics & $\begin{array}{c}\text { Sensitivity } \\
\text { number }\end{array}$ \\
\hline Amikacin & 183 & 91.50 \\
Meropenem & 157 & 78.50 \\
Piperacillin/Tazobactam & 155 & 77.50 \\
Tobramycin & 149 & 74.50 \\
Piperacillin & 139 & 69.50 \\
Ciprofloxacin & 139 & 69.50 \\
Levofloxacin & 135 & 67.50 \\
Ceftazidime & 103 & 51.50 \\
\hline
\end{tabular}


samples $(61.5 \%)$ followed by pus and wound swabs (35\%). However, study by Jayarajan et al. reported $71.37 \%$ from pus and wound swabs which was much higher and $14.2 \%$ from sputum which was much lower in comparision to our study. ${ }^{9}$

Most sensitive drug was found to be Amikacin (91.50\%) which was slightly higher than the observations made by Agrawal S et al. (85.23\%), and Jatan $\mathrm{S}$ et al. (84.21\%). ${ }^{7,10}$ Second most sensitive drug was found to be carbapenem (78.50\%) which was in concordance with Agrawal $\mathrm{S}$ et al. (78.33\%) while Agrawal $\mathrm{G}$ et al. reported 91.95\% which was much higher as compared to our study. ${ }^{11}$ Our study as well as study by Agrawal $\mathrm{S}$ et al. and S. Jatan et al. have shown decreasing sensitivity towards $\beta$ lactams, cephalosporins, and fluoroquinolones. Dahal $\mathrm{R}$ et al. also reported very low sensitivity towards $\beta$ lactams, cephalosporines and fluoroquinolones. ${ }^{12}$ Increasing resistant to $\beta$ lactams, cephalosporins and fluoroquinolones may be explained by its easy availability, easy route of administration and widely use of these drugs for treatment of various infections and it could also be due to the ability of bacteria conferring resistance to different groups of antibiotics associated with different resistance mechanism. Meta analysis done by Gauri et al. found an association between the use of quinolones and acquisition of MDRPA. ${ }^{13}$ From our study and other studies it has been observed that Amikacin with different percentage of sensitivity, is found to be the most sensitive drug for the treatment of MDRPA infection followed by carbapenems.

The percentage of MDRPA ranged from $18.6 \%$ by Shrestha $\mathrm{S}$ et al. to $100 \%$ by Moazami-Gaudarzi et al. and Ranjbar et al. ${ }^{14-16}$ Our study observed 34.5\% MDRPA which was comparable to above findings.
However Saderi H et al. (54.5\%), Yong-hua gao et al. (59.9\%), Bayani et al. (60\%) and Senthamarai et al. reported $(41.35 \%)$ which was much higher as compared to our study. ${ }^{17-20}$ From these studies it has been observed that MDRPA represents as high as $100 \%$ in some countries which is a very worrisome finding in terms of treatment, huge financial burden associated with high morbidity and mortality as well as wide spread of MDRPA in different countries. Though very limited drugs are available for the treatment of MDRPA infection at present, there seems a hope as there are some drugs in the pipeline: imipenem-relebactam, cefepimezidebactam, and murepavadin which could be the next option for the treatment of MDRPA infection. ${ }^{21}$ Although our study is a single centric study with limited number of sample size, it is expected that it shall help shed some light in the academic world of infectious diseases.

\section{CONCLUSIONS}

Multi drug resistant $P$. aeruginosa infection is constantly increasing worldwide as a major cause of nosocomial infection. Indiscriminate use of antimicrobials has led to develop resistance to many groups of antimicrobials, thus leaving clinicians with less therapeutic options for the treatment of MDRPA infection. Therefore, it is important to carry out such studies in large scale to formulate antimicrobial policy in the hospital as well as at the national level. Implementation of antimicrobial stewardship and infection control policies in hospitals can minimise the spread of MDRPA. In addition, national strategic approach is required for the use of antimicrobials which can preserve the effective drugs for the future use.

To cite this article: Bhatta S, Pradhan M, Singh A, Chaudhary R, Singh YI. Antimicrobial sensitivity pattern of Pseudomonas aeruginosa isolated from a tertiary care hospital. MJSBH. 2020;19(2):70-4.

Conflict of Interest: None declared

\section{REFERENCES}

1. Pier GB, Ramphal R. Pseudomonas aeruginosa. In: Mandell GL,Bennet JE. Dolin R.(eds) Principles And Practice Of Infectious Diseases 7th ed. Philadelphia: Churchill Livingston Elsevier; 2010. p.2835-60. DOI: https://doi.org/ 10.1016/B978-0-443-06839-3.00219-8

2. Lister PD, Wolter DJ, Hanson ND. Antibacterial-Resistant Pseudomonas aeruginosa: Clinical Impact and Complex Regulation of Chromosomally Encoded Resistance Mechanisms. 2009. Clin Microbiol Rev. 2009;22(4):582-610. DOI: https://doi.org/10.1128/CMR.00040-09 
3. Morrison AJ, Wenzel RP. Epidemiology of infection due to Pseudomonas aeruginosa. Rev. Infect. Dis. 1984;6(3): 627-42. DOI: https://doi.org/10.1093/clinids/6.Supplement_3.S627

4. Emori TG, Culver DH, Horan TC, Jarvis WR, White JW, Olson DR, et al. National nosocomial infections surveillance system (NNIS): description of surveillance methods. Am J Infect. 1991;19(1):19-35. DOI: https:// doi.org/10.1016/0196-6553(91)90157-8

5. Surveillance (NNIS) System report, data summary from January 1992 through June 2004, issued October 2004. Am J Infect. Control 32:470-85. DOI: https://doi.org/10.1111/j.1469-0691.2011.03570.x

6. Clinical and Laboratory Standards Institute. Performance standards for antimicrobial disc susceptibility testing; Approved standard, CLSI document M100 -S29, 29th informational supplement, CLSI, Wayne, PA; 2019.

7. Magiorakos AP, Srinivasan A, Carey R, Carmeli Y, Falagas ME, Giske CG, et al. Multidrug-resistant, extensively drug-resistant and pan-drug-resistant bacteria: an international expert proposal for interim standard definitions for acquired resistance. Clin Microbiol Infect. 2012;18(3):268-81. DOI: https://doi.org/10.1111/j. 1469-0691.2011.03570.x

8. Agarwal S, Rai R, Karnaker V, Sanjeev H, Pai Asha KB, Ganesh HR. Prevalence and antimicrobial susceptibility pattern of clinical isolates of Pseudomonas aeruginosa in a tertiary care teaching hospital. Intl J Appl Biol Pharmac Tech. 2013;4(3):251-3.

9. Ansari S, Dhital R, Shrestha S, Thapa S, Puri R, Chaudhary N et al. Growing Menace of Antibacterial Resistance in Clinical Isolates of Pseudomonas aeruginosa in Nepal: An Insight of Beta-Lactamase Production. Biomed res int. 2016;2016:6437208. DOI: http://dx.doi.org/10.1155/2016/6437208

10. Jayarajan D, Abirami V, Dreviyaraj VMA, Akashya V. Evaluation of high multiple antibiotic resistant index and virulent factors among clinical strains of pseudomonas aeruginosa. Intl J Sci Res. 2018;9(1):598-603.

11. Sherchan J, Gurung P, Bam D, Sherchan J. Multi-Drug Resistant Bacterial Strains in Lower Respiratory Tract Infections, Antibiotic Sensitivity Patterns and Risk Factors. NJST. 2013;13(1):157-63. DOI:https://doi.org/10.3126/ njst.v13i1.7454

12. Agrawal G, Lodhi RB, Kamalakar UP, Khadse RK, Jalgaonkar SV. Study of metallo- $\beta$-lactamase production in clinical isolates of Pseudomonas aeruginosa. Ind J Med Microbiol. 2008;26(4):349-51 DOI: $10.4103 / 0255-0857.43573$

13. Dahal RH, Chaudhary DK. Microbial Infections and Antimicrobial Resistance in Nepal: Current Trends and Recommendations. Open Microbiol J. 2018;12:230-42. DOI: 10.2174/1874285801812010230

14. Raman G, Avendano EE, Chan J, Merchant S, Puzniak L. Risk factors for hospitalised patients with resistant or multidrug-resistant Pseudomonas aeruginosa infections: a systematic review and meta-analysis. Antimicrob Resist Infect Control. 2018;7:79. DOI: https://doi.org/10.1186/s13756-018-0370-9

15. Shrestha S, Amatya R, Adhikari RP. Prevalence and antibiogram of pseudomonas aeruginosa isolated from clinical specimens in a Teaching Hospital, Kathmandu. Intl J Infec Dis. 2016;45(1):115-16. DOI: https://doi.org/10.1016/ j.ijid.2016.02.292

16. Moazami-Goudarzi S, Eftekhar F. Assessment of Carbapenem Susceptibility and Multidrug-Resistance in Pseudomonas aeruginosa Burn Isolates in Tehran. Jundishapur J Microbiol. 2013;6(2):162-65. DOI: 10.5812/jjm. 5036

17. Ranjabar R, Owlia P, Saderi H, Mansouri S, Jonaidi-Jafari N, Izadi M . Characterisation of Psedomonas aeruginosa from burned patients hospitalised in a major burn center in a Tehran, Iran. Acts Med Iran. 2011:49(10):675-9. PMID: 22071644

18. Horieh Saderi, Parviz Owlia. Detection of Multidrug Resistant (MDR) and Extremely Drug Resistant (XDR) Pseudomonas Aeruginosa Isolated from Patients in Tehran, Iran. Iran J Pathol. 2015;10(4):265-71. PMID: 26351496

19. Gao Y, Guan W, Zhu Y, Chen R, Zhang G. Antibiotic-resistant Pseudomonas aeruginosa infection in patients with bronchiectasis: Prevalence, risk factors and prognostic implications. Intl J COPD. 2018;13:237-46. DOI: https:// doi.org/10.2147/COPD.S150250

20. Bayani M, Siadati S,Rajabnia R, Taher AA .Drug resistance of Pseudomonas aeruginosa and Enterobacter cloacae isolated from ICU,Babol, Northern Iran. Int J Mol Cell Med. 2013;2(4):204-9. PMID: 24551814

21. Senthamarai S. Resistance pattern of Pseudomonas aeruginosa in a tertiary care hospital of Kanchipuram, Tamilnadu, India. JCDR. 2014;8(5):30-2. DOI: 10.7860/JCDR/2014/7953.4388 
22. Horcajada JP, Montero M, Oliver A, Sorlí L, Luque S, Gómez-Zorrilla S, et al. Epidemiology and treatment of multidrug-resistant and extensively drug-resistant Pseudomonas aeruginosa infections. Clin Microbiol Rev. 2019;32(4):00031-19. DOI: 10.1128/CMR.00031-19 